\title{
COVID-19 Vaccination Reactogenicity in Persons With Multiple Sclerosis
}

Farren Basil Shaw Briggs, PhD, ScM, Farrah J. Mateen, MD, PhD, Hollie Schmidt, MS, Keisha M. Currie, MRC, CRC, Heather M. Siefers, MS, Slavka Crouthamel, MBA, Bruce F. Bebo, PhD, Julie Fiol, MSW, BSN, RN, MSCN, Michael K. Racke, MD, Kevin C. O'Connor, PhD, Laura G. Kolaczkowski, BA, Phyllis Klein, PhD, RN, Sara Loud, MS, MBA, and Robert Nicholas McBurney, PhD

Neurol Neuroimmunol Neuroinflamm 2022;9:e1104. doi:10.1212/NXI.0000000000001104

\section{Abstract}

\section{Background and Objectives}

There are limited data on severe acute respiratory syndrome coronavirus 2 (SARS-CoV-2) vaccine reactogenicity in persons with multiple sclerosis (PwMS) and how reactogenicity is affected by disease-modifying therapies (DMTs). The objective of this retrospective crosssectional study was to generate real-world multiple sclerosis-specific vaccine safety information, particularly in the context of specific DMTs, and provide information to mitigate specific concerns in vaccine hesitant PwMS.

\section{Methods}

Between 3/2021 and 6/2021, participants in iConquerMS, an online people-powered research network, reported SARS-CoV-2 vaccines, experiences of local (itch, pain, redness, swelling, or warmth at injection site) and systemic (fever, chills, fatigue, headache, joint pain, malaise, muscle ache, nausea, allergic, and other) reactions within 24 hours (none, mild, moderate, and severe), DMT use, and other attributes. Multivariable models characterized associations between clinical factors and reactogenicity.

\section{Results}

In 719 PwMS, 64\% reported experiencing a reaction after their first vaccination shot, and 17\% reported a severe reaction. The most common reactions were pain at injection site (54\%), fatigue $(34 \%)$, headache $(28 \%)$, and malaise (21\%). Younger age, being female, prior SARSCoV-2 infection, and receiving the ChAdOx1 nCoV-19 (Oxford-AstraZeneca) vs BNT162b2 (Pfizer-BioNTech) vaccine were associated with experiencing a reaction after the first vaccine dose. Similar relationships were observed for a severe reaction, including higher odds of reactions among PwMS with more physical impairment and lower odds of reactions for PwMS on an alpha4-integrin blocker or sphingosine-1-phosphate receptor modulator. In 442 PwMS who received their second vaccination shot, $74 \%$ reported experiencing a reaction, whereas $22 \%$ reported a severe reaction. Reaction profiles after the second shot were similar to those reported after the first shot. Younger PwMS and those who received the mRNA-1273 (Moderna) vs BNT162b2 vaccine reported higher reactogenicity after the second shot, whereas those on a sphingosine-1-phosphate receptor modulator or fumarate were significantly less likely to report a reaction.

\section{Discussion}

SARS-CoV-2 vaccine reactogenicity profiles and the associated factors in this convenience sample of PwMS appear similar to those reported in the general population. PwMS on specific
Correspondence

Dr. Briggs

farren.briggs@case.edu

\section{MORE ONLINE}

COVID-19 Resources

For the latest articles, invited commentaries, and blogs from physicians around the world

NPub.org/COVID 19 


\section{Glossary}

COVID-19 = coronavirus disease 2019; DMT = disease-modifying therapy; PDDS = Patient-Determined Disease Steps; PwMS = persons with multiple sclerosis; PPRN = people-powered research network; SARS-CoV-2 = severe acute respiratory syndrome coronavirus 2 .

DMTs were less likely to report vaccine reactions. Overall, the short-term vaccine reactions experienced in the study population were mostly self-limiting, including pain at the injection site, fatigue, headache, and fever.

Preventing severe acute respiratory syndrome coronavirus 2 (SARS-CoV-2) infection, the cause of coronavirus disease 2019 (COVID-19), is the most pressing global health crisis. ${ }^{1}$ Since December 2020, several SARS-CoV-2 vaccines have been developed and available to varying degrees across the globe, including novel mRNA (i.e., Pfizer-BioNTech [BNT162b2] and Moderna [mRNA-1273]) and viral vector (i.e., Johnson \& Johnson's Janssen [Ad26.COV2.S] and Oxford-AstraZeneca [ChAdOx1 nCoV-19]) vaccines. These vaccines have demonstrated high safety and efficacy in preventing SARS-CoV-2 infection and severe COVID-19. ${ }^{2-5}$ However, safety data from vaccine trials in individuals with autoimmune conditions, including persons with multiple sclerosis (PwMS), have not been reported or may not exist. In particular, BNT162b2 trials excluded individuals with autoimmune diseases and those treated with immunomodulatory therapies; mRNA-1273 trials excluded those treated with immunomodulatory therapies within 6 months of entry. Considering the novel vaccine strategies deployed and the uncertain impact of immunomodulatory therapies, vaccine safety data in PwMS are of great interest, including short-term reactogenicity. This is especially important because $>20 \%$ of PwMS may be vaccine hesitant, ${ }^{6,7}$ with specific concerns related to vaccine safety, vaccine efficacy, and potential side effects. ${ }^{6,8}$ Among vaccine-hesitant PwMS, shortterm side effects were a major concern for $26 \%$ and a minor concern for $49 \%$ of individuals. ${ }^{8}$

The reported reactogenicity of BNT162b2, mRNA-1273, Ad26.COV2.S, and ChAdOx1 $\mathrm{nCoV}-19$ from vaccine trials and observational studies of the general population has been characterized by transient and mild to moderate symptoms, including pain at the injection site, headache, fatigue, fever/ chills, and myalgia. ${ }^{2,5,9,10}$ On average, a higher proportion of women, younger adults, and those with a prior SARS-CoV-2 infection report experiencing these short-term symptoms, and reactogenicity is elevated for the specific vaccines (i.e., higher reactogenicity in mRNA-1273 compared with BNT162b2) and for the second vaccine shot for vaccination protocols requiring 2 shots. ${ }^{4,9,11,12}$

To date, a few observational studies have begun to characterize vaccine safety profiles in PwMS. Eighty-one Dutch patients with MS, alongside patients with rheumatoid arthritis, experienced a similar burden of transient local and systemic vaccine reactions that were primarily self-limiting in comparison to unaffected controls when accounting for age, sex, and vaccine type. ${ }^{13}$ In a study of 555 Israeli patients with MS, descriptive statistics qualitatively suggested higher vaccine reactogenicity in younger patients, patients with less physical impairment, and patients treated with a disease-modifying therapy (DMT). However, formal statistical comparisons, including comparisons accounting for putative confounders (i.e., age, sex, vaccine type, disability, and prior SARS-CoV-2 infection) were not conducted. ${ }^{14}$ In a third study of 425 Italian patients with MS, 55\% of PwMS who self-reported being on a DMT reported an early BNT162b2 vaccine reaction, whereas 63\% of PwMS not on a DMT reported an early BNT162b2 reaction $(p=0.07)$ - however, this comparison also did not account for putative confounders nor were specific DMT classes investigated. ${ }^{15}$ Thus, the sociodemographic and clinical attributes independently associated with vaccine reactogenicity in PwMS remain incompletely characterized, and the impact of specific classes of DMTs, which have diverse mechanisms of action, remains largely unknown.

Here, we report findings from a cohort of $>700$ PwMS who received a SARS-CoV-2 vaccine and were members of the iConquerMS people-powered research network (PPRN). ${ }^{16}$ PwMS reported acute local and systemic reactions experienced within 24 hours of vaccination, and relationships between self-reported attributes (e.g., physical impairment and DMT status) and reactogenicity were examined in multivariable models. Our primary objective was to generate realworld MS-specific vaccine safety information for PwMS and health care professionals, particularly in the context of specific DMTs, and provide information that may help mitigate specific concerns in vaccine hesitant PwMS.

\section{Methods}

\section{Ethics Board Approval and Informed Consent}

Since October 2014, an independent ethics review board (WCG IRB, formerly Copernicus Group IRB) has approved the website content, survey instruments, informed consent, and collateral materials for the iConquerMS PPRN. Survey instruments specific for this study were approved by the WCG IRB. All participants electronically provided informed consent before study enrollment. The analysis of deidentified data was deemed research not involving human subjects by the Case Western Reserve University IRB (IRB No.: STUDY20210761). 


\section{Study Population and Study Design}

iConquerMS and the National MS Society promoted the launch of this study through their electronic mailing lists and webinars. PwMS who were or subsequently registered in the iConquerMS PPRN, an online research network, were invited to participate in this study. iConquerMS is an initiative of the Accelerated Cure Project for MS, developed in collaboration with the Complex Adaptive Systems Initiative at Arizona State University, its information technology services partner, Ordinal Data, Inc., and with the communications firm, Ogilvy. ${ }^{16}$ iConquerMS emphasizes governance by PwMS and is supported by an integrated information technology and communications platform featuring an online portal (iConquerMS.org) through which stakeholders can participate in and drive MS research.

Beginning March 22, 2021, consenting iConquerMS members were invited to complete surveys about their experiences regarding their SARS-CoV-2 vaccination(s), including vaccine reactions after their first and second shots (if applicable) and other sociodemographic and clinical attributes. By June 9, 2021, 825 study participants, who self-reported having had diagnoses of MS or clinically isolated syndrome administered by a neurologist or other physician, had completed the surveys as a part of this retrospective cross-sectional study.

\section{Exclusion Criteria and Data Quality Control}

We excluded participants who reported invalid dates for their first vaccination (preceding December 16, 2020, or after June 8, $2021 ; n=31)$, who did not report their age $(n=3)$, who were aged $<18$ years $(n=1)$, who were unsure whether they had a vaccine reaction $(n=13)$, or who did not know their DMT status $(\mathrm{n}=8)$. We excluded 48 of these remaining 769 participants because we could not determine their DMT status at vaccination or because they gave an invalid date or a date after their vaccination for their last DMT dose. Of the remaining 721 participants, 2 subjects were excluded due to inconsistencies in their reporting of the manufacturer of their first and second vaccines. As a result, the final study population for analyses related to the first vaccination shot consisted of 719 iConquerMS participants (87.1\% of the source population). Sixtyfive percent $(n=466)$ of these participants had received a second vaccine shot; however, 15 participants reported invalid dates for their second vaccination in relationship to their first vaccination (i.e., receiving the second shot on the same day as the first shot). We excluded 8 participants who were unsure whether they had a reaction to their second vaccinations. Therefore, the final study population for analyses related to the second vaccination shot consisted of 442 iConquerMS participants (53.6\% of the source population).

\section{SARS-CoV-2 Vaccine Reactions}

Participants provided dates of their first and second SARS$\mathrm{CoV}-2$ vaccinations and vaccine manufacturer name. They were then prompted to report reactions experienced within the 24 hours after their first and second vaccine administrations. Participants could have answered none, mild, moderate, or severe to the following injection site (local) reactions: itch; pain, soreness, or tenderness; redness; swelling; or warmth. They were also asked about any systemic reactions, including chills; fatigue; fever/feeling feverish; headache; joint pain; malaise; muscle ache (other than at the injection site); nausea; immediate allergic reaction (i.e., rash, swelling, difficulty breathing, fast heartbeat, dizziness, or fainting); or any other reaction. We generated 4 binary outcomes: (1) experiencing any reaction after the first shot (any mild, moderate, or severe reaction vs none); (2) experiencing any severe reaction after the first shot (any severe reaction vs all other responses [mild, moderate, or none]); (3) experiencing any reaction after the second shot (if applicable); and (4) experiencing any severe reaction after the second short (if applicable).

\section{MS Treatment Status at Vaccination}

Participants provided their recent DMT histories, including DMT and date of last administration. Using a rubric for anticipated impact of a DMT (see eMethods, links.lww.com/NXI/ A651), we determined that for 23 participants who reported being on a DMT, their last treatment date was far outside of the recommended treatment frequency guidelines. These 23 were coded as not actively on a DMT at vaccination (see eMethods, links.lww.com/NXI/A651). A categorical variable was constructed for DMT status at vaccination: $0=$ no DMT or not actively on a DMT; 1 = B-cell depletion therapies (Kesimpta, Ocrevus, and Rituxan); $2=\mathrm{T} / \mathrm{B}$-cell proliferation inhibitor (Aubagio); 3 = interferon beta drugs (Avonex, Betaseron, Plegridy, and Rebif); 4 = glatiramer acetate (Copaxone and Glatopa); $5=$ sphingosine-1-phosphate receptor (S1PR) modulators (Gilenya, Mayzent, and Zeposia); 6 = alpha-4integrin blocker (Tysabri); $7=$ other immune cell regulators (Lemtrada and Mavenclad); and $8=$ fumarates (Tecfidera and Vumerity).

\section{Other Covariates}

Age, sex, disease duration (time from the first symptom suggestive of MS to present), subtype (relapsing-remitting, secondary progressive, primary progressive, and clinically isolated syndrome), Patient-Determined Disease Steps (PDDS), Latinx ethnicity, race (White, Black, and Other/Mixed), vaccine manufacturer (BNT162b2 [Pfizer-BioNTech], mRNA-1273 [Moderna], Ad26.COV2.S [Johnson \& Johnson's Janssen], ChAdOxl nCoV-1 [Oxford-AstraZeneca], and other), and prior self-reported SARS-CoV-2 infection (yes/no) were also reported. Indicator variables were generated to capture missing/ unknown responses for ethnicity (0.7\%), race (1.4\%), disease subtype (1.5\%), and prior SARS-CoV-2 infection (1.3\%) to maximize the number of observations retained across analyses.

\section{Statistical Analyses}

Descriptive statistics were generated for the entire study population and stratified by reaction status after the first and, if present, second vaccination shots, including mean and SD for continuous measures and percentages for categorical measures. Multivariable logistic regressions were used to examine the relationships (ORs; 95\% CIs) between DMT status and all other covariates with the following outcomes: (1) any 
Table 1 Attributes of the Study Population Who Received Their First Vaccine Shot

\begin{tabular}{|c|c|c|c|c|c|}
\hline \multicolumn{2}{|l|}{ Attribute (mean [SD] or \%) } & All MS cases & $\begin{array}{l}\text { MS cases } \\
\text { reporting } \\
\text { no reaction }\end{array}$ & $\begin{array}{l}\text { MS cases } \\
\text { reporting any } \\
\text { reaction }\end{array}$ & $\begin{array}{l}\text { MS cases reporting } \\
\text { any severe reaction }\end{array}$ \\
\hline \multicolumn{2}{|l|}{$\mathbf{N}$} & 719 & $260(36.2 \%)$ & $459(63.8 \%)$ & $122(16.9 \%)$ \\
\hline \multicolumn{2}{|l|}{ Age (yr) } & $53.0(11.8)$ & $55.8(11.6)$ & $51.4(11.6)$ & $50.8(11.2)$ \\
\hline \multicolumn{2}{|l|}{ Female } & $84.6 \%(n=608)$ & $79.6 \%(n=207)$ & $87.4 \%(n=401)$ & $91.8 \%(n=112)$ \\
\hline \multicolumn{2}{|l|}{ Latinx } & $4.7 \%(n=34)$ & $3.5 \%(n=9)$ & $5.5 \%(n=25)$ & $12.3 \%(n=15)$ \\
\hline \multirow[t]{3}{*}{ Race } & White & $94.2 \%(n=677)$ & $91.9 \%(n=239)$ & $95.4 \%(n=438)$ & $94.3 \%(n=115)$ \\
\hline & Non-White & $4.4 \%(n=32)$ & $6.2 \%(n=16)$ & $3.5 \%(n=16)$ & $4.1 \%(n=5)$ \\
\hline & Unknown & $1.4 \%(n=10)$ & $1.9 \%(n=5)$ & $1.1 \%(n=5)$ & $1.6 \%(n=2)$ \\
\hline \multirow[t]{5}{*}{ Subtype } & Relapsing-remitting & $70.0 \%(n=593)$ & $63.5 \%(n=165)$ & $73.6 \%(n=338)$ & $75.4 \%(n=92)$ \\
\hline & Secondary progressive & $17.5 \%(n=126)$ & $19.6 \%(n=51)$ & $16.3 \%(n=75)$ & $15.6 \%(n=19)$ \\
\hline & Primary progressive & $9.2 \%(n=66)$ & $13.5 \%(n=35)$ & $6.7 \%(n=31)$ & $5.7 \%(n=7)$ \\
\hline & Clinically isolated syndrome & $1.8 \%(n=13)$ & $1.5 \%(n=4)$ & $2.0 \%(n=9)$ & $0.8 \%(n=1)$ \\
\hline & Unknown & $1.5 \%(n=11)$ & $1.9 \%(n=5)$ & $1.3 \%(n=6)$ & $2.5 \%(n=3)$ \\
\hline \multicolumn{2}{|l|}{ Disease duration } & $13.8(9.2)$ & $15.3(10.3)$ & $13.0(8.5)$ & $13.0(8.3)$ \\
\hline \multicolumn{2}{|c|}{ Patient-Determined Disease Steps } & $2.5(2.2)$ & $2.8(2.3)$ & $2.3(2.2)$ & $2.6(2.0)$ \\
\hline \multirow[t]{3}{*}{ Prior COVID-19 infection } & No & $92.2 \%(n=663)$ & $95.8 \%(n=248)$ & $90.2 \%(n=414)$ & $80.3 \%(n=98)$ \\
\hline & Yes & $6.5 \%(n=47)$ & $3.1 \%(n=9)$ & $8.5 \%(n=39)$ & $17.2 \%(n=21)$ \\
\hline & Unsure & $1.3 \%(n=9)$ & $1.1 \%(n=3)$ & $1.3 \%(n=6)$ & $2.5 \%(n=3)$ \\
\hline \multirow[t]{5}{*}{ Vaccine manufacturer } & BNT162b2 (Pfizer-BioNTech) & $56.9 \%(n=409)$ & $59.2 \%(n=154)$ & $55.6 \%(n=255)$ & $54.1 \%(n=66)$ \\
\hline & mRNA-1273 (Moderna) & $35.9 \%(n=258)$ & $34.2 \%(n=89)$ & $36.8 \%(n=169)$ & $38.5 \%(n=47)$ \\
\hline & $\begin{array}{l}\text { Ad26.COV2.S (Johnson } \\
\text { \& Johnson's Janssen) }\end{array}$ & $4.3 \%(n=31)$ & $5.4 \%(n=14)$ & $3.7 \%(n=17)$ & $2.5 \%(n=3)$ \\
\hline & $\begin{array}{l}\text { ChAdOx1 nCoV-1 } \\
\text { (Oxford-AstraZeneca) }\end{array}$ & $2.8 \%(n=20)$ & $0.8 \%(n=2)$ & $3.9 \%(n=18)$ & $4.9 \%(n=6)$ \\
\hline & Other & $0.1 \%(n=1)$ & $0.4 \%(n=1)$ & $0 \%(n=0)$ & $0 \%(n=0)$ \\
\hline \multirow{9}{*}{$\begin{array}{l}\text { Disease-modifying } \\
\text { therapy at vaccination }\end{array}$} & Not actively on a DMT & $25.0 \%(n=180)$ & $28.5 \%(n=74)$ & $23.1 \%(n=106)$ & $26.2 \%(n=32)$ \\
\hline & $\begin{array}{l}\text { B-cell depleters (Kesimpta, } \\
\text { Ocrevus, and Rituxan) }\end{array}$ & $27.3 \%(n=196)$ & $26.9 \%(n=70)$ & $27.5 \%(n=126)$ & $30.3 \%(n=37)$ \\
\hline & $\begin{array}{l}\text { T/B-cell proliferation inhibitor } \\
\text { (Aubagio) }\end{array}$ & $4.7 \%(n=34)$ & $5.4 \%(n=14)$ & $4.4 \%(n=20)$ & $4.9 \%(n=6)$ \\
\hline & $\begin{array}{l}\text { Interferon betas (Avonex, } \\
\text { Betaseron, Plegridy, and Rebif) }\end{array}$ & $6.3 \%(n=45)$ & $4.2 \%(n=11)$ & $7.4 \%(n=34)$ & $7.4 \%(n=9)$ \\
\hline & $\begin{array}{l}\text { Glatiramer acetate } \\
\text { (Copaxone and Glatopa) }\end{array}$ & $7.2 \%(n=52)$ & $7.7 \%(n=20)$ & $7.0 \%(n=32)$ & $8.2 \%(n=10)$ \\
\hline & $\begin{array}{l}\text { S1P receptor modulator } \\
\text { (Gilenya, Mayzent, and Zeposia) }\end{array}$ & $7.8 \%(n=56)$ & $9.6 \%(n=25)$ & $6.8 \%(n=31)$ & $3.3 \%(n=4)$ \\
\hline & Alpha4-integrin blocker (Tysabri) & $7.2 \%(n=52)$ & $5.8 \%(n=15)$ & $8.1 \%(n=37)$ & $3.3 \%(n=4)$ \\
\hline & $\begin{array}{l}\text { Other immune cell regulators } \\
\text { (Lemtrada and Mavenclad) }\end{array}$ & $2.4 \%(n=17)$ & $2.7 \%(n=7)$ & $2.2 \%(n=10)$ & $3.3 \%(n=4)$ \\
\hline & Fumarates (Tecfidera and Vumerity) & $12.1 \%(n=87)$ & $9.2 \%(n=24)$ & $13.7 \%(n=63)$ & $13.1 \%(n=16)$ \\
\hline
\end{tabular}

Abbreviations: COVID-19 = coronavirus disease 2019; DMT = disease-modifying therapy; PwMS = persons with multiple sclerosis. 
reaction after the first shot; (2) any severe reaction after the first shot; (3) any reaction after the second shot; and (4) any severe reaction after the second shot. For the latter 2 outcomes, we also adjusted for experiencing any reaction after the first vaccination.

We conducted a sensitivity analysis restricted to participants who were US residents, and we explored stratified models for experiencing any local reactions, any severe local reactions, any systemic reactions, or any severe systemic reactions after the first and second vaccinations, respectively. All analyses were conducted in Stata v13.1 (StataCorp LP, College Station, TX). A 2-sided alpha of 0.05 was considered statistically significant.

\section{Data Availability}

Data related to this study are available from iConquerMS. Interested parties must obtain approval from the iConquerMS Research Committee, that consists of PwMS and other MS stakeholders, in addition to an ethics board approval and a completed data transfer agreement between the Accelerated Cure Project and the requesting institution from qualified investigators.

\section{Results}

\section{Participant Characteristics}

The final study population consisted of $719 \mathrm{PwMS}$ with a mean age of 53 years ( $\mathrm{SD}=12$ years) and who were predominantly non-Latinx (95\%), White (94\%), female (85\%), and US residents (90\%) (Table 1). Most participants had relapsingremitting MS (70\%), followed by secondary progressive MS (18\%), primary progressive MS (9\%), and clinically isolated syndrome $(2 \%)$, with a mean disease duration of 14 years $(\mathrm{SD}=9)$ and moderate disability (mean PDDS $=2.5)$. A prior SARS-CoV-2 infection was reported by $7 \%$ of participants. A quarter of the study population were not actively on a DMT, whereas $27 \%$ were on B cell-depleting therapies, $12 \%$ were on fumarates, $8 \%$ were on S1PR modulators, $7 \%$ were on glatiramer acetate, $7 \%$ were on alpha4-integrin blocker, $6 \%$ were on interferons, $5 \%$ were on Lemtrada or Mavenclad, and less than $<3 \%$ were on other DMTs. The attributes of participants who had their second vaccination were similar to the overall study population $(\mathrm{N}=442$; Table 2).

\section{Vaccination Experiences}

Most PwMS received the BNT162b2 (Pfizer-BioNTech) vaccine (57\%), followed by the mRNA-1273 (Moderna) vaccine (36\%), the Ad26.COV2.S (Johnson \& Johnson's Janssen) vaccine (4\%), and ChAdOx1 nCoV-1 (Oxford-AstraZeneca) (3\%) vaccines. Among participants who received their second vaccination (Table 2), most received the BNT162b2 vaccine (61\%), followed by the mRNA-1273 vaccine (38\%) and then the ChAdOx $1 \mathrm{nCoV}-1$ vaccine $(1 \%)$ - and $>90 \%$ received their second vaccine within a month of their first vaccine (Table 2).
Nearly two-thirds (64\%) of the study population reported experiencing any reaction and $17 \%$ reported any severe reaction within 24 hours of the first vaccination shot $(\mathrm{N}=719$; Table 1). The most common reactions were pain at injection site $(54 \%)$, fatigue $(34 \%)$, headache $(28 \%)$, and malaise (21\%) (Figure 1A; eTable 1, links.lww.com/NXI/A651). All other symptoms were experienced by $<20 \%$ of the study population, including $<3 \%$ who self-reported an allergic reaction to their vaccine.

Vaccine reactogenicity was elevated after the second vaccine shot, with $74 \%$ of the study population reporting any reaction and $22 \%$ reporting any severe reaction $(\mathrm{N}=442$; Table 2$)$. Similar reaction profiles were reported after the second shot, and the most common reactions were pain at injection site (61\%), fatigue (53\%), headache (40\%), malaise (35\%), muscle ache (32\%), and chills (26\%) (Figure 1B; eTable 2, links.lww.com/NXI/A651). A notable observation was that $82 \%$ of participants with a severe reaction after their second shot had a reaction after their first shot (Table 2).

\section{Associations With Vaccine Reactions}

In a multivariable model for experiencing any reaction within 24 hours after the first vaccine shot, adjusting for likely confounders (Table 3), participants were less likely to report having a reaction for each additional 1-year increase in age $(\mathrm{OR}=0.98$; 95\% CI: 0.96-0.99; $p=0.007)$. Non-White participants were less likely to report experiencing a vaccine reaction compared with White participants ( $\mathrm{OR}=0.4$; 95\% CI: $0.19-0.86$; $p=$ 0.018 ), and there was a nonsignificant trend for fewer reactions among participants on S1PR modulators compared with those who were not actively on a DMT $(\mathrm{OR}=0.50 ; 95 \% \mathrm{CI}$ : 0.26-1.0; $p=0.051$; Figure 1, C and E; eTable 1, links.lww. com/NXI/A651). Women with MS were significantly more likely to report a vaccine reaction compared with men with MS (OR $=1.89$; 95\% CI: 1.21-2.97; $p=0.0054)$, as were those with a prior SARS-CoV-2 infection vs those without $(\mathrm{OR}=3.38$; 95\% CI: 1.5-7.6; $p=0.0032$ ), and those who received a ChAdOx1 $\mathrm{nCoV}-1$ vs a $\mathrm{BNT} 162 \mathrm{~b} 2$ vaccine $(\mathrm{OR}=6.57 ; 95 \%$ CI: $1.43-30.16$; $p=0.015$ ).

In a multivariable model for experiencing any severe reaction after the first vaccine shot (Table 3), similar associations were observed for age (OR $=0.97$; 95\% CI: 0.95-0.99; $p=0.012)$, women $(\mathrm{OR}=2.41 ; 95 \% \mathrm{CI}: 1.16-4.99 ; p=0.018)$, prior SARS-CoV-2 infection (OR $=5.45 ; 95 \%$ CI: 2.78-10.67; $\left.p=8 \times 10^{-7}\right)$, a ChAdOx1 nCoV-1 vaccine compared with $\mathrm{BNT} 162 \mathrm{~b} 2$ vaccine $(\mathrm{OR}=3.25 ; 95 \% \mathrm{CI}: 1.09-9.7 ; p=0.034)$, and being on S1PR modulators vs no DMT (OR $=0.21$; $95 \%$ CI: $0.06-0.71 ; p=0.012$; Figure $1, \mathrm{C}$ and E; eTable 1, links. lww.com/NXI/A651). Novel associations were that Latinx participants were more likely to report severe reactions compared with non-Latinx participants ( $\mathrm{OR}=3.95 ; 95 \% \mathrm{CI}$ : $\left.1.8-8.65 ; 6 \times 10^{-4}\right)$; disabled participants reported were also more likely to report severe reactions per unit increase in PDDS (OR $=1.17$; 95\% CI: $1.03-1.33 ; p=0.013)$; and participants on alpha4-integrin blockers were much less likely to 
Table 2 Attributes of the Study Population Who Received Their Second Vaccine Shot

\begin{tabular}{|c|c|c|c|c|c|}
\hline \multicolumn{2}{|l|}{ Attribute (mean [SD] or \%) } & All MS cases & $\begin{array}{l}\text { MS cases } \\
\text { reporting } \\
\text { no reaction }\end{array}$ & $\begin{array}{l}\text { MS cases } \\
\text { reporting any } \\
\text { reaction }\end{array}$ & $\begin{array}{l}\text { MS cases reporting } \\
\text { any severe reaction }\end{array}$ \\
\hline \multicolumn{2}{|l|}{$\mathbf{N}$} & 442 & $115(26.0 \%)$ & $327(74.0 \%)$ & $99(22.4 \%)$ \\
\hline \multicolumn{2}{|l|}{ Age (yr) } & $53.5(12.2)$ & $59.1(9.5)$ & $51.5(12.4)$ & $51.7(11.3)$ \\
\hline \multicolumn{2}{|l|}{ Female } & $83.9 \%(n=371)$ & $76.5 \%(n=88)$ & $86.5 \%(n=283)$ & $88.9 \%(n=88)$ \\
\hline \multicolumn{2}{|l|}{ Latinx } & $4.6 \%(n=21)$ & $3.5 \%(n=4)$ & $5.2 \%(n=17)$ & $5.1 \%(n=5)$ \\
\hline \multirow[t]{3}{*}{ Race } & White & $94.6 \%(n=419)$ & $93.9 \%(n=108)$ & $95.1 \%(n=311)$ & $96.0 \%(n=95)$ \\
\hline & Non-White & $4.8 \%(n=21)$ & $5.2 \%(n=6)$ & $4.0 \%(n=13)$ & $2.0 \%(n=2)$ \\
\hline & Unknown & $0.7 \%(n=3)$ & $0.9 \%(n=1)$ & $0.9 \%(n=3)$ & $2.0 \%(n=2)$ \\
\hline \multirow[t]{5}{*}{ Subtype } & Relapsing-remitting & $68.3 \%(n=302)$ & $62.6 \%(n=72)$ & $70.3 \%(n=230)$ & $64.7 \%(n=64)$ \\
\hline & Secondary progressive & $18.3 \%(n=81)$ & $19.1 \%(n=22)$ & $18.0 \%(n=59)$ & $22.2 \%(n=22)$ \\
\hline & Primary progressive & $10.2 \%(n=45)$ & $15.7 \%(n=19)$ & $8.3 \%(n=27)$ & $10.1 \%(n=10)$ \\
\hline & Clinically isolated syndrome & $1.1 \%(n=5)$ & $0.9 \%(n=1)$ & $1.2 \%(n=4)$ & $1.0 \%(n=1)$ \\
\hline & Unknown & $2.0 \%(n=9)$ & $1.7 \%(n=2)$ & $2.1 \%(n=7)$ & $2.0 \%(n=2)$ \\
\hline \multicolumn{2}{|l|}{ Disease duration } & $13.9(9.1)$ & $16.1(8.8)$ & $13.1(9.1)$ & $13.7(9.7)$ \\
\hline \multicolumn{2}{|c|}{ Patient-Determined Disease Steps } & $2.5(2.3)$ & $2.9(2.3)$ & $2.4(2.3)$ & $2.6(2.3)$ \\
\hline \multirow[t]{3}{*}{ Prior COVID-19 infection } & No & $91.6 \%(n=405)$ & $89.6 \%(n=103)$ & $92.4 \%(n=302)$ & $87.9 \%(n=87)$ \\
\hline & Yes & $7.0 \%(n=31)$ & $8.7 \%(n=10)$ & $6.4 \%(n=21)$ & $10.1 \%(n=10)$ \\
\hline & Unsure & $1.4 \%(n=6)$ & $1.7 \%(n=2)$ & $1.2 \%(n=4)$ & $2.0 \%(n=2)$ \\
\hline \multirow[t]{5}{*}{ Vaccine manufacturer } & BNT162b2 (Pfizer-BioNTech) & $60.9 \%(n=269)$ & $71.3 \%(n=82)$ & $57.2 \%(n=187)$ & $52.5 \%(n=52)$ \\
\hline & mRNA-1273 (Moderna) & $37.6 \%(n=166)$ & $26.1 \%(n=30)$ & $41.6 \%(n=136)$ & $46.5 \%(n=46)$ \\
\hline & $\begin{array}{l}\text { Ad26.COV2.S (Johnson } \\
\text { \& Johnson's Janssen) }\end{array}$ & $0 \%(n=0)$ & $0 \%(n=0)$ & $0 \%(n=0)$ & $0 \%(n=0)$ \\
\hline & $\begin{array}{l}\text { ChAdOx1 nCoV-1 } \\
\text { (Oxford-AstraZeneca) }\end{array}$ & $1.4 \%(n=6)$ & $1.7 \%(n=2)$ & $1.2 \%(n=4)$ & $1.0 \%(n=1)$ \\
\hline & Other & $0.2 \%(n=1)$ & $0.9 \%(n=1)$ & $0 \%(n=0)$ & $0 \%(n=0)$ \\
\hline \multirow{9}{*}{$\begin{array}{l}\text { Disease-modifying } \\
\text { therapy at vaccination }\end{array}$} & Not actively on a DMT & $24.9 \%(n=110)$ & $29.6 \%(n=34)$ & $23.2 \%(n=76)$ & $27.3 \%(n=27)$ \\
\hline & $\begin{array}{l}\text { B-cell depleters (Kesimpta, } \\
\text { Ocrevus, and Rituxan) }\end{array}$ & $28.5 \%(n=126)$ & $22.6 \%(n=26)$ & $30.6 \%(n=100)$ & $28.3 \%(n=28)$ \\
\hline & T/B-cell proliferation inhibitor (Aubagio) & $5.0 \%(n=22)$ & $7.0 \%(n=8)$ & $4.3 \%(n=14)$ & $3.0 \%(n=3)$ \\
\hline & $\begin{array}{l}\text { Interferon betas (Avonex, } \\
\text { Betaseron, Plegridy, and Rebif) }\end{array}$ & $6.8 \%(n=30)$ & $4.4 \%(n=5)$ & $7.7 \%(n=25)$ & $9.1 \%(n=9)$ \\
\hline & Glatiramer acetate (Copaxone and Glatopa) & $7.9 \%(n=35)$ & $5.2 \%(n=6)$ & $8.9 \%(n=29)$ & $9.1 \%(n=9)$ \\
\hline & $\begin{array}{l}\text { S1P receptor modulator } \\
\text { (Gilenya, Mayzent, and Zeposia) }\end{array}$ & $7.5 \%(n=33)$ & $12.2 \%(n=14)$ & $5.8 \%(n=19)$ & $2.0 \%(n=2)$ \\
\hline & Alpha4-integrin blocker (Tysabri) & $7.9 \%(n=35)$ & $7.0 \%(n=8)$ & $8.3 \%(n=27)$ & $10.1 \%(n=10)$ \\
\hline & $\begin{array}{l}\text { Other immune cell regulators } \\
\text { (Lemtrada and Mavenclad) }\end{array}$ & $1.6 \%(n=7)$ & $0.9 \%(n=1)$ & $1.8 \%(n=6)$ & $2.0 \%(n=2)$ \\
\hline & Fumarates (Tecfidera and Vumerity) & $10.0 \%(n=44)$ & $11.3 \%(n=13)$ & $9.5 \%(n=31)$ & $9.1 \%(n=9)$ \\
\hline \multicolumn{2}{|c|}{ Any reaction to the first vaccine shot } & $63.4 \%(n=280)$ & $25.2 \%(n=29)$ & $76.8 \%(n=251)$ & $81.8 \%(n=81)$ \\
\hline \multicolumn{2}{|c|}{ Any severe reaction to the first vaccine shot } & $15.4 \%(n=68)$ & $7.8 \%(n=9)$ & $18.1 \%(n=59)$ & $39.4 \%(n=39)$ \\
\hline \multicolumn{2}{|c|}{ Received second vaccine within $31 \mathrm{~d}$ of the first vaccine } & $91.9 \%(n=406)$ & $93.0 \%(n=107)$ & $91.4 \%(n=299)$ & $95.0 \%(n=94)$ \\
\hline
\end{tabular}

Abbreviations: COVID-19 = coronavirus disease 2019; DMT = disease-modifying therapy; PwMS = persons with multiple sclerosis. 


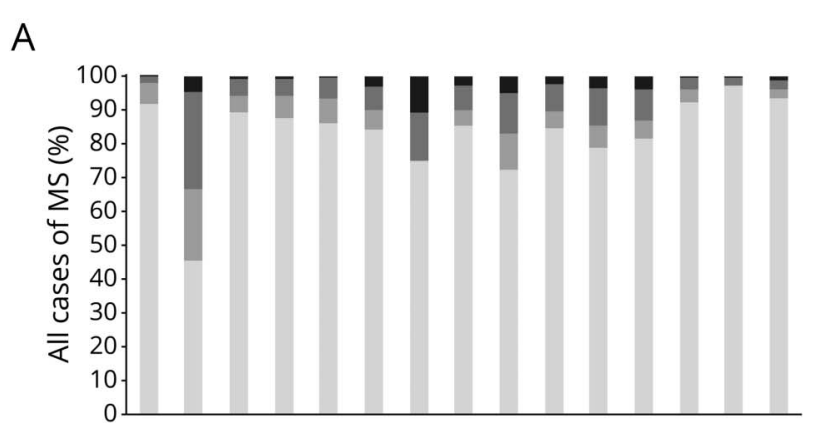

C

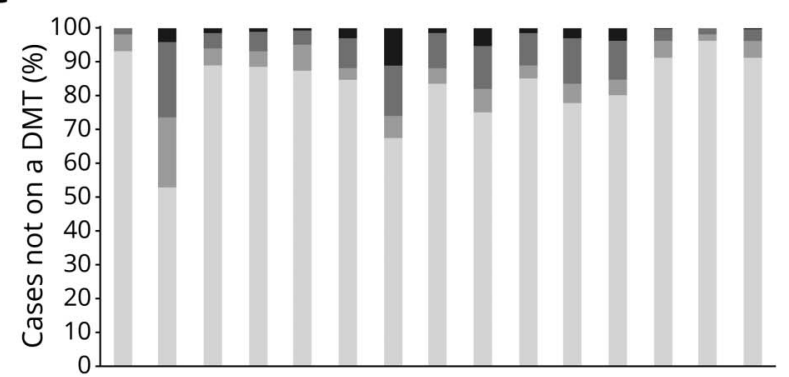

E

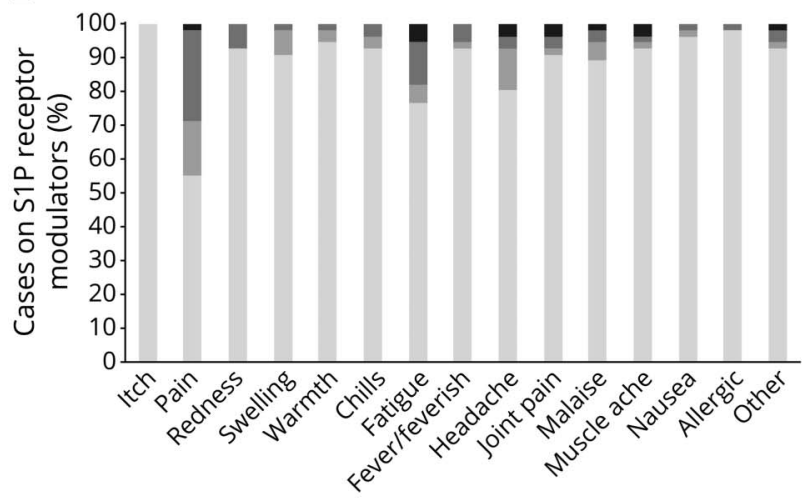

First vaccine shot reactions

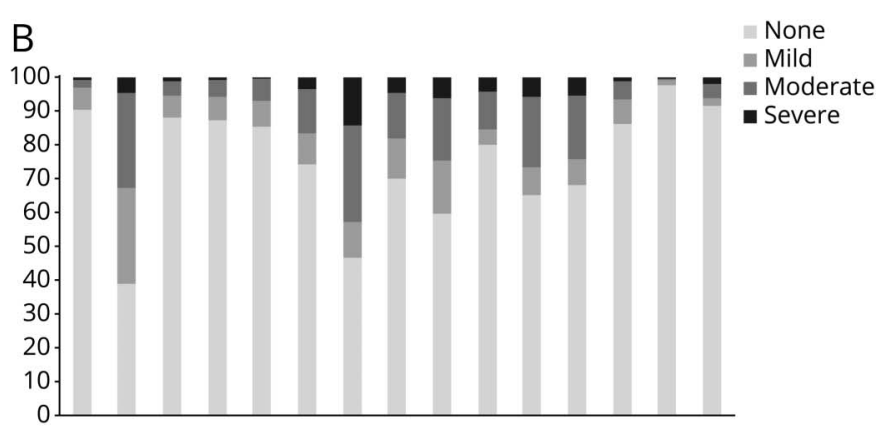

D

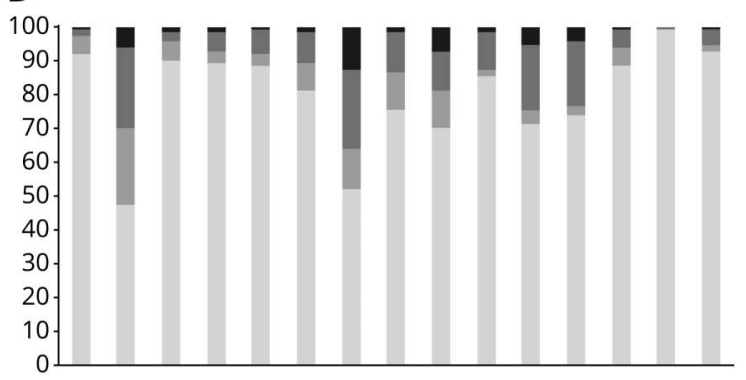

$\mathrm{F}$

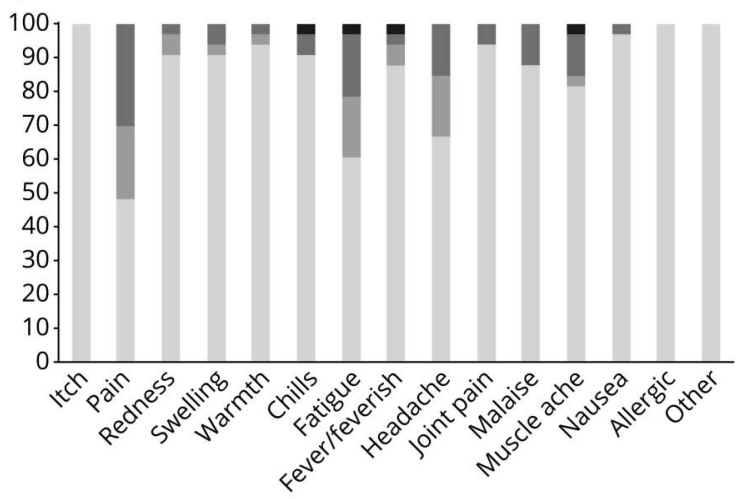

Second vaccine shot reactions report experiencing severe reactions compared with those not actively on a DMT (OR $=0.25$; 95\% CI: $0.08-0.83 ; p=0.023)$.

In the multivariable models for experiencing any reaction or any severe reaction after the second vaccine shot, similar associations were observed across models (Table 4). Older participants were less likely to report any reaction $(\mathrm{OR}=0.93$; 95\% CI: $0.9-0.96 ; p=9.5 \times 10^{-6}$ ) or any severe reaction $(\mathrm{OR}=0.97 ; 95 \% \mathrm{CI}: 0.95-1.00 ; p=0.025)$ after the second shot. Participants on S1PR modulators (OR $=0.18$; 95\% CI: 0.06-0.58; $p=0.0055$; Figure 1, D and F; eTable 2, links.lww. com/NXI/A651) or fumarates (OR $=0.31 ; 95 \% \mathrm{CI}$ : 0.11-0.83; $p=0.027$ ) were less likely to report experiencing any reaction compared with those not actively on a DMT, whereas only those on S1PR modulators were less likely to report any severe reactions after the second shot $(\mathrm{OR}=0.15$; 95\% CI: 0.03-0.73; $p=0.019$; Figure 1, D and F; eTable 2, links.lww.com/NXI/A651). Participants who received the
mRNA-1273 vaccine had increased odds of experiencing any reaction $\left(\mathrm{OR}=2.62 ; 95 \% \mathrm{CI}: 1.46-4.71 ; p=8.7 \times 10^{-4}\right)$ or any severe reaction $(\mathrm{OR}=1.69 ; 95 \% \mathrm{CI}$ : $1.03-2.75 ; p=0.037)$ after the second shot compared with those who received the BNT162b2 vaccines. Furthermore, individuals who experienced a reaction after their first shot had substantially elevated odds of experiencing any reaction $(\mathrm{OR}=12.42 ; 95 \% \mathrm{CI}$ : 6.99-22.06; $\left.p=7.7 \times 10^{-16}\right)$ or any severe reaction $(\mathrm{OR}=3.15$; 95\% CI: $\left.1.71-5.78 ; p=2.2 \times 10^{-5}\right)$ after their second shot.

Across all models, there were no significant associations for subtype or disease duration. Similar associations were observed across models when restricting to US residents and when stratifying by local or systemic reactions (data not shown). A curious observation was that participants on interferons were more likely to report any local reaction after the first vaccine shot compared with those not actively on a DMT (OR = 2.2; 95\% CI: 1.04-4.84; $p=0.04$ ), but the association did not persist across other models. 
Table 3 Multivariable Logistic Regression Associations for Any Reaction or Any Severe Reaction After the First Vaccine Shot

\begin{tabular}{|c|c|c|c|c|c|}
\hline & & \multicolumn{2}{|l|}{ Any reaction } & \multicolumn{2}{|c|}{ Any severe reaction } \\
\hline \multicolumn{2}{|l|}{ Covariate (mean [SD] or \%) } & OR $(95 \% \mathrm{CI})$ & $p$ Value & OR $(95 \% \mathrm{CI})$ & $p$ Value \\
\hline \multicolumn{2}{|l|}{$\mathbf{N}^{\mathrm{a}}$} & 718 & & 718 & \\
\hline \multicolumn{2}{|l|}{ Age (yr) } & $0.98(0.96,0.99)$ & 0.0070 & $0.97(0.95,0.99)$ & 0.012 \\
\hline \multicolumn{2}{|l|}{ Female } & $1.89(1.21,2.97)$ & 0.0054 & $2.41(1.16,4.99)$ & 0.018 \\
\hline \multicolumn{2}{|l|}{ Latinx } & $1.36(0.59,3.12)$ & 0.47 & $3.95(1.8,8.65)$ & $6.0 \times 10^{-4}$ \\
\hline \multirow[t]{3}{*}{ Race } & White & 1 & - & 1 & - \\
\hline & Non-White & $0.4(0.19,0.86)$ & 0.018 & $0.94(0.32,2.7)$ & 0.90 \\
\hline & Unknown & $0.66(0.15,2.88)$ & 0.58 & $0.86(0.14,5.4)$ & 0.88 \\
\hline \multirow[t]{5}{*}{ Subtype } & Relapsing-remitting & 1 & - & 1 & - \\
\hline & Secondary progressive & $1.06(0.62,1.79)$ & 0.84 & $0.58(0.28,1.17)$ & 0.13 \\
\hline & Primary progressive & $0.61(0.32,1.15)$ & 0.13 & $0.4(0.15,1.04)$ & 0.061 \\
\hline & Clinically isolated syndrome & $0.77(0.22,2.71)$ & 0.69 & $0.15(0.01,1.72)$ & 0.13 \\
\hline & Unknown & $0.86(0.23,3.16)$ & 0.82 & $1.56(0.35,6.92)$ & 0.56 \\
\hline \multicolumn{2}{|l|}{ Disease duration } & $0.98(0.96,1)$ & 0.11 & $1(0.97,1.03)$ & 0.82 \\
\hline \multicolumn{2}{|c|}{ Patient-Determined Disease Steps } & $0.99(0.9,1.09)$ & 0.908 & $1.17(1.03,1.33)$ & 0.013 \\
\hline \multirow[t]{3}{*}{ Prior COVID-19 infection } & No & 1 & - & 1 & - \\
\hline & Yes & $3.38(1.5,7.6)$ & 0.0032 & $5.45(2.78,10.67)$ & $8.0 \times 10^{-7}$ \\
\hline & Unsure & $1.19(0.28,5.16)$ & 0.81 & $4.22(0.91,19.64)$ & 0.067 \\
\hline \multirow[t]{4}{*}{ Vaccine manufacturer } & BNT162b2 (Pfizer-BioNTech) & 1 & - & 1 & - \\
\hline & mRNA-1273 (Moderna) & $1.2(0.85,1.7)$ & 0.30 & $1.19(0.76,1.86)$ & 0.45 \\
\hline & Ad26.COV2.S (Johnson \& Johnson's Janssen) & $0.88(0.4,1.93)$ & 0.74 & $0.57(0.16,2.07)$ & 0.39 \\
\hline & ChAdOx1 nCoV-1 (Oxford-AstraZeneca) & $6.57(1.43,30.16)$ & 0.015 & $3.25(1.09,9.7)$ & 0.034 \\
\hline \multirow{9}{*}{$\begin{array}{l}\text { Disease-modifying } \\
\text { therapy at vaccination }\end{array}$} & Not actively on a DMT & 1 & - & 1 & - \\
\hline & B-cell depleters (Kesimpta, Ocrevus, and Rituxan) & $0.92(0.58,1.48)$ & 0.74 & $0.87(0.48,1.59)$ & 0.65 \\
\hline & T/B-cell proliferation inhibitor (Aubagio) & $0.74(0.34,1.64)$ & 0.46 & $0.91(0.33,2.54)$ & 0.86 \\
\hline & Interferon betas (Avonex, Betaseron, Plegridy, and Rebif) & $1.69(0.77,3.73)$ & 0.19 & $1.11(0.45,2.73)$ & 0.82 \\
\hline & Glatiramer acetate (Copaxone and Glatopa) & $0.83(0.42,1.65)$ & 0.60 & $0.99(0.42,2.33)$ & 0.97 \\
\hline & S1P receptor modulator (Gilenya, Mayzent, and Zeposia) & $0.51(0.26,1)$ & 0.051 & $0.21(0.06,0.71)$ & 0.012 \\
\hline & Alpha4-integrin blocker (Tysabri) & $1.25(0.59,2.62)$ & 0.56 & $0.25(0.08,0.83)$ & 0.023 \\
\hline & Other immune cell regulators (Lemtrada and Mavenclad) & $0.45(0.15,1.33)$ & 0.15 & $0.71(0.19,2.59)$ & 0.60 \\
\hline & Fumarates (Tecfidera and Vumerity) & $1.27(0.69,2.34)$ & 0.44 & $0.85(0.41,1.8)$ & 0.68 \\
\hline
\end{tabular}

Abbreviations: COVID-19 = coronavirus disease 2019; DMT = disease-modifying therapy; PwMS = persons with multiple sclerosis.

a 718 of 719 PwMS were retained in these models because there was only 1 PwMS who received their vaccine from another manufacturer; therefore, there were insufficient data to retain this observation.

\section{Discussion}

The SARS-CoV-2 vaccine is highly recommended for PwMS. ${ }^{17}$ However, $\sim 6 \%$ of American PwMS are unwilling to receive the SARS-CoV-2 vaccine, and $>20 \%$ of PwMS are vaccine hesitant. ${ }^{6,7}$ Frequent concerns for vaccine hesitant PwMS were vaccine safety and efficacy and potential shortand long-term side effects. ${ }^{6,8}$ In addition, most vaccine hesitant PwMS expressed the desire for additional information. ${ }^{8}$ Unfortunately, no safety data pertaining specifically to PwMS 
Table 4 Multivariable Logistic Regression Associations for Any Reaction or Any Severe Reaction After the Second Vaccine Shot

\begin{tabular}{|c|c|c|c|c|c|}
\hline \multirow{2}{*}{ Covariate (mean [SD] or \%) } & & \multicolumn{2}{|l|}{ Any reaction } & \multicolumn{2}{|c|}{ Any severe reaction ${ }^{a}$} \\
\hline & & OR $(95 \% \mathrm{Cl})$ & $p$ Value & OR $(95 \% \mathrm{CI})$ & $p$ Value \\
\hline \multicolumn{2}{|l|}{$\mathbf{N}^{\mathbf{b}}$} & 441 & & 441 & \\
\hline \multicolumn{2}{|l|}{ Age (yr) } & $0.93(0.9,0.96)$ & $9.5 \times 10^{-6}$ & $0.97(0.95,1)$ & 0.025 \\
\hline \multicolumn{2}{|l|}{ Female } & $1.35(0.66,2.76)$ & 0.40 & $1.54(0.74,3.22)$ & 0.25 \\
\hline \multicolumn{2}{|l|}{ Latinx } & $0.85(0.22,3.32)$ & 0.82 & - & - \\
\hline \multirow[t]{3}{*}{ Race } & White & 1 & - & 1 & - \\
\hline & Non-White & $0.96(0.25,3.64)$ & 0.95 & $0.36(0.07,1.77)$ & 0.21 \\
\hline & Unknown & $8.4(0.21,339.8)$ & 0.26 & $6.34(0.7,57.66)$ & 0.10 \\
\hline \multirow[t]{5}{*}{ Subtype } & Relapsing-remitting & 1 & - & 1 & - \\
\hline & Secondary progressive & $1.47(0.61,3.56)$ & 0.33 & $1.84(0.83,4.09)$ & 0.13 \\
\hline & Primary progressive & $0.74(0.29,1.88)$ & 0.60 & $1.64(0.63,4.24)$ & 0.31 \\
\hline & Clinically isolated syndrome & $0.55(0.03,10.78)$ & 0.69 & $0.51(0.04,5.76)$ & 0.59 \\
\hline & Unknown & $7.62(0.93,62.44)$ & 0.063 & $1.51(0.25,9.12)$ & 0.65 \\
\hline \multicolumn{2}{|l|}{ Disease duration } & $0.98(0.95,1.01)$ & 0.32 & $1.00(0.96,1.03)$ & 0.88 \\
\hline \multicolumn{2}{|c|}{ Patient-Determined Disease Steps } & $1.05(0.9,1.22)$ & 0.55 & $1.03(0.88,1.17)$ & 0.74 \\
\hline \multirow[t]{3}{*}{ Prior COVID-19 infection } & No & 1 & - & 1 & - \\
\hline & Yes & $0.53(0.18,1.51)$ & 0.19 & $2.00(0.85,4.74)$ & 0.11 \\
\hline & Unsure & $0.87(0.1,7.32)$ & 0.90 & $2.20(0.35,13.8)$ & 0.40 \\
\hline \multirow[t]{3}{*}{ Vaccine manufacturer } & BNT162b2 (Pfizer-BioNTech) & 1 & - & 1 & - \\
\hline & mRNA-1273 (Moderna) & $2.62(1.46,4.71)$ & $8.7 \times 10^{-4}$ & $1.69(1.03,2.75)$ & 0.037 \\
\hline & ChAdOx1 nCoV-1 (Oxford-AstraZeneca) & $0.26(0.04,1.72)$ & 0.16 & $0.53(0.06,4.84)$ & 0.57 \\
\hline \multirow{9}{*}{$\begin{array}{l}\text { Disease-modifying } \\
\text { therapy at vaccination }\end{array}$} & Not actively on a DMT & 1 & - & 1 & - \\
\hline & B-cell depleters (Kesimpta, Ocrevus, and Rituxan) & $0.78(0.36,1.68)$ & 0.40 & $0.61(0.31,1.21)$ & 0.16 \\
\hline & T/B-cell proliferation inhibitor (Aubagio) & $0.44(0.13,1.46)$ & 0.14 & $0.51(0.13,2.03)$ & 0.34 \\
\hline & Interferon betas (Avonex, Betaseron, Plegridy, and Rebif) & $1.06(0.28,4.02)$ & 0.99 & $1.22(0.45,3.30)$ & 0.69 \\
\hline & Glatiramer acetate (Copaxone and Glatopa) & $2.33(0.73,7.43)$ & 0.29 & $1.23(0.47,3.19)$ & 0.67 \\
\hline & S1P receptor modulator (Gilenya, Mayzent, and Zeposia) & $0.18(0.06,0.58)$ & 0.0055 & $0.15(0.03,0.73)$ & 0.019 \\
\hline & Alpha4-integrin blocker (Tysabri) & $0.47(0.14,1.52)$ & 0.15 & $1.02(0.38,2.72)$ & 0.97 \\
\hline & Other immune cell regulators (Lemtrada and Mavenclad) & $0.95(0.06,13.92)$ & 0.96 & $0.60(0.10,3.69)$ & 0.58 \\
\hline & Fumarates (Tecfidera and Vumerity) & $0.31(0.11,0.83)$ & 0.027 & $0.66(0.26,1.70)$ & 0.39 \\
\hline \multicolumn{2}{|c|}{ Any reaction to the first vaccine shot } & $12.42(6.99,22.06)$ & $7.7 \times 10^{-16}$ & $3.15(1.71,5.78)$ & $2.2 \times 10^{-4}$ \\
\hline
\end{tabular}

Abbreviations: COVID-19 = coronavirus disease 2019; DMT = disease-modifying therapy; PWMS = persons with multiple sclerosis.

${ }^{a}$ Ethnicity was excluded from the model as there was insufficient variation in this variable in relation to the outcome variable.

b 441 of 442 PwMS were retained in these models because there was only 1 PwMS who received their vaccine from another manufacturer; therefore, there were insufficient data to retain this observation.

have been reported from the various SARS-CoV-2 vaccine trials, and extrapolation of the safety data from the general population to PwMS is unclear, largely due to the uncertain impact of DMTs. We investigated the relationships between sociodemographic/clinical attributes and DMT status on short-term (24 hours) SARS-CoV-2 vaccine reactogenicity in PwMS participating in an online research network. Overall, the vaccine reaction profiles in PwMS appear similar to those 
reported for the general population. Also, factors associated with vaccine reactogenicity in the general population were similarly associated in PwMS (i.e., younger age, being female, prior SARS-CoV-2 infection, and vaccine manufacturer). Of interest, in multivariable models adjusted for likely confounders, PwMS treated with S1PR modulators were less likely to have any reaction or any severe reaction after the first and second vaccinations, whereas those treated with alpha-4integrin blockers were less likely to have any severe reaction after the first shot, and those on fumarates were less likely to have any reaction after the second shot.

In the current study population of PwMS, two-thirds reported experiencing a reaction within 24 hours after their first vaccine. This reactogenicity prevalence is comparable to those observed in the vaccine trials in the general population (e.g., $88 \%$ of mRNA-1273 recipients reported a short-term reaction).$^{2-5}$ For the 2 most common vaccines, BNT162b2 and mRNA-1273, vaccine trial participants reported a burden of pain at the injection site $(>75 \%)$, fatigue $(>35 \%)$, and headache $(>30 \%)$ after their the first vaccine dose that were at frequencies higher than those observed in the current study population of PwMS (eTable 1, links.lww.com/NXI/A651). ${ }^{2,3}$ Other reactogenicity symptoms had similar patterns for the first and second vaccine shots. Thus, our findings do not suggest that there is an excess of short-term side effects in PwMS compared with the general population after SARS-CoV-2 vaccinations. Furthermore, there were no associations between vaccine reactogenicity and MS subtype or disease duration, but PwMS with a higher PDDS may experience a slight increase in severe reactions after the first but not the second vaccination-reasons for this observation are unclear.

A key observation was the lower vaccine reactogenicity in PwMS treated with specific DMTs, but not all. For example, there was no difference in reactogenicity in PwMS treated with B-cell depleters compared with those not actively on a DMT, and results (data not shown) were similar for varied temporal windows (i.e., last treated within 12 or 24 weeks of vaccination). In MS, S1PR modulators inhibit the egression of lymphocytes from lymph nodes, ${ }^{18}$ and it is possible that this same mechanism may have resulted in PwMS experiencing fewer local and systemic vaccine reactions compared with PwMS who were not actively on a DMT (Figure 1, C-F; eTables 1 and 2, links.lww.com/NXI/A651). However, considering S1PRs are expressed throughout the body, regulating diverse cellular responses in the immune (innate and adaptive), cardiovascular, and neurologic systems, there are likely multiple plausible mechanisms contributing to lower reactogenicity in those treated with S1PR modulators. ${ }^{18}$ The mechanism of action for the alpha4-integrin blocker DMT (natalizumab) is to limit the movement of leukocytes from blood vessels into central system nervous tissues and minimize subsequent inflammation ${ }^{19}$; we can speculate that this process of sequestering leukocytes may have contributed to our observation of fewer severe reactions in PwMS after the first vaccination. In MS, the mechanisms of action for fumarates are diverse, decreasing subsets of lymphocytes (i.e., $\mathrm{CD}^{+}$and $\mathrm{CD} 8^{+} \mathrm{T}$ cells) and creating a bias toward anti-inflammatory immune cells in blood $^{20}$; this promotion of an anti-inflammatory environment may have contributed in part to the observed lower burden of vaccine reactions in PwMS after the second vaccine. The precise mechanisms through which these DMTs result in diminished reactogenicity are not clear, much less the potential relationships between vaccine reactogenicity and immunogenicity in the context of specific DMTs. We do note that a similar pattern of diminished SARS-CoV-2 reactogenicity has been reported in individuals with inflammatory bowel disease treated with advanced immunomodulatory therapies. ${ }^{21}$ Thus, we can speculate that individuals with other autoimmune conditions and treated with similar immunomodulatory therapies may have similar reactogenicity profiles.

The current study has several strengths, including being able to examine the relationships between SARS-CoV-2 vaccine reactogenicity and specific DMTs, and we importantly accounted for the effects of likely confounders. Second, we present findings based on real-world data collected in $>700$ PwMS who primarily resided across the entire United States (there was also a small number of PwMS from other countries), and data collection was not dependent on in-person visits to a health care provider. Third, attributes of our study population appear representative of the general MS population; however, the study population were older on average and primarily non-Latinx Whites. Fourth, we were able to consider reactogenicity for multiple vaccines in relation to one another and most importantly consider relationships for all major DMT classes.

Our primary limitation was the potential impact of selection bias in this convenience cohort of iConquerMS participants who might not represent all individuals with MS (i.e., PwMS with limited internet access; non-White PwMS) and was unlikely to have included PwMS who experienced serious adverse events (i.e., serious life-threatening or extended hospitalization events). As a result, no definitive conclusions on the prevalence of adverse reactions or vaccine withdrawals can be made. Another limitation is the absence of an internal nonMS population to allow for direct comparisons and that all information was based on self-report; however, we noted a few instances of inconsistent data reporting and addressed these issues through careful data quality control. Furthermore, considering the historic nature of receiving a SARS-CoV-2 vaccine, concerns with quality of vaccine reactogenicity recall are diminished. It is also possible that some reactions were overlooked due to the structure of the surveys; however, participants were able to enter free text describing other symptoms experienced (e.g., $0.8 \%$ and $0.7 \%$ of participants reported unexplained diarrhea after their first and second vaccinations, respectively). Another limitation is that participants could have enrolled several weeks before or after receiving their first vaccine dose-although this flexibility in 
participation is a strength, it may also introduce information bias (distribution of reactogenicity by enrollment is presented in eTable 3, links.lww.com/NXI/A651); however, results did not change when adjusting for time between enrollment and first vaccine shot, nor was the coefficient for this variable significant across models (data not shown). A final limitation is the modest response rate by iConquerMS participants active in the 2 years prior the current study (<25\%; eMethods, links.lww.com/NXI/A651)-however, demographic attributes are similar between the current study population and recently active iConquerMS participants who were not a part of these analyses (eMethods, links. lww.com/NXI/A651).

In summary, we hope that the experiences of PwMS in this cohort can inform vaccine-hesitant PwMS and provide important information to health care providers, particularly those in countries where vaccine accessibility is still limited. We provide evidence that that short-term safety profiles of SARS-CoV-2 vaccines in PwMS are similar to those reported in vaccine trials for general population and that individuals treated with specific classes of DMT are less likely to experience short-term reactogenicity. Overall, the short-term vaccine reactions experienced in this cohort of PwMS were mostly self-limiting, including pain at the injection site, fatigue, headache, and fever, providing a reassuring picture for future PwMS who are yet to be vaccinated.

\section{Acknowledgment}

The authors acknowledge Dr. Nina Bozinov, who provided critical feedback.

\section{Study Funding}

National Multiple Sclerosis Society (Grant SI-2103-37386).

\section{Disclosure}

iConquerMS is an initiative of the Accelerated Cure Project for Multiple Sclerosis (ACP). ACP has received grants, collaboration funding, payments for use of assets, or in-kind contributions from the following companies: EMD Serono, Sanofi/Genzyme, Biogen, Genentech, AbbVie, Octave, GlycoMinds, Pfizer, MedDay, AstraZeneca, Teva, Mallinckrodt, MSDx, Regeneron Genetics Center, BC Platforms, and Celgene. ACP has also received funding from the PatientCentered Outcomes Research Institute and the National Multiple Sclerosis Society (NMSS). Farren Briggs has received research grants from the NMSS, the Michael J. Fox Foundation, and the National Institute for Health (NIH). F.J. Mateen has received research grants from Biogen, Sumaira Foundation, NMSS, and NIH. She participated as a site investigator in clinical trials through IQVIA (Genentech, EMD Serono) and has served on ad hoc advisory boards for Horizon Therapeutics, Genentech, and Biogen. Hollie Schmidt has nothing to disclose. K.M. Currie has served as an advisor for Novartis and Janssen. H.M. Siefers and S. Crouthamel have nothing to disclose. B.F. Bebo and J. Fiol are employed by the NMSS. Michael Racke is employed by Quest
Diagnostics. K.C. O'Connor has received research support from Ra Pharma, now a part of UCB Pharma, and Alexion, now part of AstraZenca, and is a consultant and equity shareholder of Cabaletta Bio. He is the recipient of a sponsored research subaward from the University of Pennsylvania, the primary financial sponsor of which is Cabaletta Bio. He has served as consultant/advisor for Alexion Pharmaceuticals, and for Roche, and he has received speaking fees from Alexion and Viela Bio, now part of Horizon Therapeutics. L.G. Kolaczkowski has served as a patient advisor for EMD Serono, EMD Merck, Novartis, Merakoi, Janssen/Johnson \& Johnson, and Genentech. P. Klein and S. Loud report no disclosures. R.N. McBurney has received consulting payments from EMD Serono, which have been donated to ACP. Go to Neurology.org/NN for full disclosures.

\section{Publication History}

Received by Neurology: Neuroimmunology \& Neuroinflammation August 7, 2021. Accepted in final form September 21, 2021.

Appendix Authors

\begin{tabular}{lll}
\hline Name & Location & Contribution \\
\hline $\begin{array}{l}\text { Farren Basil } \\
\text { Phaw Briggs, }\end{array}$ & $\begin{array}{l}\text { Department of Population } \\
\text { and Quantitative Health } \\
\text { Science, School of } \\
\text { Medicine, Cleveland, OH }\end{array}$ & $\begin{array}{l}\text { Drafting/revision of the } \\
\text { manuscript for content, } \\
\text { including medical writing } \\
\text { for content; study concept } \\
\text { or design; and analysis or } \\
\text { interpretation of data }\end{array}$ \\
$\begin{array}{l}\text { Farrah J. } \\
\text { Mateen, MD, } \\
\text { PhD }\end{array}$ & $\begin{array}{l}\text { Department of Neurology, } \\
\text { Massachusetts General }\end{array}$ & $\begin{array}{l}\text { Drafting/revision of the } \\
\text { manuscript for content, } \\
\text { including medical writing } \\
\text { for content; study concept } \\
\text { or design; and analysis or }\end{array}$ \\
& & $\begin{array}{l}\text { interpretation of data } \\
\text { ind }\end{array}$
\end{tabular}

Hollie Accelerated Cure Project Drafting/revision of the Schmidt, MS for MS, Waltham, MA manuscript for content, including medical writing for content; major role in the acquisition of data; study concept or design; and analysis or interpretation of data

\begin{tabular}{|c|c|c|}
\hline $\begin{array}{l}\text { Keisha M. } \\
\text { Currie, MRC, } \\
\text { CRC }\end{array}$ & $\begin{array}{l}\text { Currie Consultancy, LLC } \\
\text { Eastover, SC }\end{array}$ & $\begin{array}{l}\text { Drafting/revision of the } \\
\text { manuscript for content, } \\
\text { including medical writing } \\
\text { for content, and study } \\
\text { concept or design }\end{array}$ \\
\hline $\begin{array}{l}\text { Heather M. } \\
\text { Siefers, MS }\end{array}$ & $\begin{array}{l}\text { International AIDS Vaccine } \\
\text { Initiative, Frederick, MD }\end{array}$ & $\begin{array}{l}\text { Drafting/revision of the } \\
\text { manuscript for content, } \\
\text { including medical writing } \\
\text { for content, and study } \\
\text { concept or design }\end{array}$ \\
\hline $\begin{array}{l}\text { Slavka } \\
\text { Crouthamel, } \\
\text { MBA }\end{array}$ & $\begin{array}{l}\text { Mammoth Hospital, } \\
\text { Mammoth Lakes, CA }\end{array}$ & $\begin{array}{l}\text { Drafting/revision of the } \\
\text { manuscript for content, } \\
\text { including medical writing } \\
\text { for content, and study } \\
\text { concept or design }\end{array}$ \\
\hline $\begin{array}{l}\text { Bruce F. Bebo, } \\
\text { PhD }\end{array}$ & $\begin{array}{l}\text { National Multiple Sclerosis } \\
\text { Society }\end{array}$ & $\begin{array}{l}\text { Drafting/revision of the } \\
\text { manuscript for content, } \\
\text { including medical writing } \\
\text { for content; study concept } \\
\text { or design; and analysis or } \\
\text { interpretation of data }\end{array}$ \\
\hline
\end{tabular}


Appendix (continued)

\begin{tabular}{|c|c|c|}
\hline Name & Location & Contribution \\
\hline $\begin{array}{l}\text { Julie Fiol, } \\
\text { MSW, BSN, RN, } \\
\text { MSCN }\end{array}$ & $\begin{array}{l}\text { National Multiple Sclerosis } \\
\text { Society }\end{array}$ & $\begin{array}{l}\text { Drafting/revision of the } \\
\text { manuscript for content, } \\
\text { including medical writing } \\
\text { for content, and study } \\
\text { concept or design }\end{array}$ \\
\hline $\begin{array}{l}\text { Michael K. } \\
\text { Racke, MD }\end{array}$ & $\begin{array}{l}\text { Medical Affairs, Quest } \\
\text { Diagnostics, Secaucus, NJ }\end{array}$ & $\begin{array}{l}\text { Drafting/revision of the } \\
\text { manuscript for content, } \\
\text { including medical writing } \\
\text { for content, and analysis or } \\
\text { interpretation of data }\end{array}$ \\
\hline $\begin{array}{l}\text { Kevin C. } \\
\text { O'Connor, PhD }\end{array}$ & $\begin{array}{l}\text { Departments of Neurology } \\
\text { and Immunobiology, Yale } \\
\text { University School of } \\
\text { Medicine, New Haven, СT }\end{array}$ & $\begin{array}{l}\text { Drafting/revision of the } \\
\text { manuscript for content, } \\
\text { including medical writing } \\
\text { for content, and analysis or } \\
\text { interpretation of data }\end{array}$ \\
\hline $\begin{array}{l}\text { Laura G. } \\
\text { Kolaczkowski, } \\
\text { BA }\end{array}$ & iConquerMS, Waltham, MA & $\begin{array}{l}\text { Drafting/revision of the } \\
\text { manuscript for content, } \\
\text { including medical writing } \\
\text { for content; major role in } \\
\text { the acquisition of data; and } \\
\text { study concept or design }\end{array}$ \\
\hline
\end{tabular}

Phyllis Klein, iConquerMS, Waltham, MA Drafting/revision of the PhD, RN manuscript for content, including medical writing for content, and study concept or design
Sara Loud, MS, Accelerated Cure Project

MBA for MS, Waltham, MA

\section{Drafting/revision of the} manuscript for content, including medical writing for content; major role in the acquisition of data; study concept or design; and analysis or interpretation of data

\begin{tabular}{lll}
\hline Robert & Accelerated Cure Project & Drafting/revision of the \\
Nicholas & for MS, Waltham, MA & manuscript for content, \\
McBurney, & & including medical writing \\
PhD & for content; major role in \\
& the acquisition of data; \\
& study concept or design; \\
& and analysis or \\
& interpretation of data
\end{tabular}

\section{References}

1. Miller IF, Becker AD, Grenfell BT, Metcalf CJE. Disease and healthcare burden of COVID-19 in the United States. Nat Med. 2020;26(8):1212-1217.

2. Polack FP, Thomas SJ, Kitchin N, et al. Safety and efficacy of the BNT162b2 mRNA Covid-19 vaccine. N Engl J Med. 2020;383(27):2603-2615.

3. Baden LR, El Sahly HM, Essink B, et al. Efficacy and safety of the mRNA-1273 SARSCoV-2 vaccine. N Engl J Med. 2021;384(5):403-416.

4. SadoffJ, Gray G, Vandebosch A, et al. Safety and efficacy of single-dose Ad26.COV2.S vaccine against Covid-19. N Engl J Med. 2021;384(23):2187-2201.

5. Voysey M, Clemens SAC, Madhi SA, et al. Safety and efficacy of the ChAdOx1 nCoV19 vaccine (AZD1222) against SARS-CoV-2: an interim analysis of four randomised controlled trials in Brazil, South Africa, and the UK. Lancet. 2021;397(10269):99-111.

6. Xiang XM, Hollen C, Yang Q, Brumbach BH, Spain RI, Wooliscroft L. COVID-19 vaccination willingness among people with multiple sclerosis. Mult Scler J Exp Transl Clin. 2021;7(2):20552173211017159.

7. Ehde DM, Roberts MK, Herring TE, Alschuler KN. Willingness to obtain COVID-19 vaccination in adults with multiple sclerosis in the United States. Mult Scler Relat Disord. 2021;49:102788.

8. Ehde DM, Roberts MK, Humbert AT, Herring TE, Alschuler KN. COVID-19 vaccine hesitancy in adults with multiple sclerosis in the United States: a follow up survey during the initial vaccine rollout in 2021. Mult Scler Relat Disord. 2021;54:103163.

9. Gee J, Marquez P, Su J, et al. First month of COVID-19 vaccine safety monitoring United States, December 14, 2020-January 13, 2021. MMWR Morb Mortal Wkly Rep $2021 ; 70(8): 283-288$

10. Shay DK, Gee J, Su JR, et al. Safety monitoring of the Janssen (Johnson \& Johnson) COVID-19 vaccine - United States, March-April 2021. MMWR Morb Mortal Wkly Rep. 2021;70(18):680-684.

11. Chapin-Bardales J, Gee J, Myers T. Reactogenicity following receipt of mRNA-based COVID-19 vaccines. JAMA. 2021;325(21):2201-2202.

12. Ebinger JE, Fert-Bober J, Printsev I, et al. Antibody responses to the BNT162b2 mRNA vaccine in individuals previously infected with SARS-CoV-2. Nat Med. 2021, 27(6):981-984.

13. Boekel L, Kummer LY, van Dam KPJ, et al. Adverse events after first COVID-19 vaccination in patients with autoimmune diseases. Lancet Rheumatol. 2021;3(8):e542-e545.

14. Achiron A, Dolev M, Menascu S, et al. COVID-19 vaccination in patients with multiple sclerosis: what we have learnt by February 2021. Mult Scler. 2021;27(6):864-870.

15. Lotan I, Wilf-Yarkoni A, Friedman Y, Stiebel-Kalish H, Steiner I, Hellmann MA Safety of the BNT162b2 COVID-19 vaccine in multiple sclerosis: early experience from a tertiary MS center in Israel. Eur J Neurol 2021;28(11):3742-3748.

16. Nowell WB, Merkel PA, McBurney RN, et al. Patient-powered research networks of the autoimmune research collaborative: rationale, capacity, and future directions. Patient. 2021;14:699-710.

17. National Multiple Sclerosis Society. Accessed July 27, 2021. nationalmssociety.org/coronavirus-covid-19-information/multiple-sclerosis-and-coronavirus/covid-19-vaccine-guidance.

18. Chaudhry BZ, Cohen JA, Conway DS. Sphingosine 1-phosphate receptor modulators for the treatment of multiple sclerosis. Neurotherapeutics. 2017;14(4):859-873.

19. Selewski DT, Shah GV, Segal BM, Rajdev PA, Mukherji SK. Natalizumab (Tysabri). AJNR Am J Neuroradiol. 2010;31(9):1588-1590.

20. Mills EA, Ogrodnik MA, Plave A, Mao-Draayer Y. Emerging understanding of the mechanism of action for dimethyl fumarate in the treatment of multiple sclerosis. Front Neurol. 2018;9:5

21. Botwin GJ, Li D, Figueiredo J, et al. Adverse events after SARS-CoV-2 mRNA vaccination among patients with inflammatory bowel disease. Am J Gastroenterol. 2021 116(8):1746-1751 


\section{Neurology \\ Neuroimmunology \& Neuroinflammation}

COVID-19 Vaccination Reactogenicity in Persons With Multiple Sclerosis Farren Basil Shaw Briggs, Farrah J. Mateen, Hollie Schmidt, et al.

Neurol Neuroimmunol Neuroinflamm 2022;9;

DOI 10.1212/NXI.0000000000001104

This information is current as of November 9, 2021

Updated Information \& Services

References

Subspecialty Collections

Permissions \& Licensing

Reprints including high resolution figures, can be found at: http://nn.neurology.org/content/9/1/e1104.full.html

This article cites 20 articles, 1 of which you can access for free at: http://nn.neurology.org/content/9/1/e1104.full.html\#\#ref-list-1

This article, along with others on similar topics, appears in the following collection(s):

All epidemiology

http://nn.neurology.org//cgi/collection/all_epidemiology COVID-19

http://nn.neurology.org//cgi/collection/covid_19

Multiple sclerosis

http://nn.neurology.org//cgi/collection/multiple_sclerosis

Public health

http://nn.neurology.org//cgi/collection/public_health

Information about reproducing this article in parts (figures,tables) or in its entirety can be found online at:

http://nn.neurology.org/misc/about.xhtml\#permissions

Information about ordering reprints can be found online:

http://nn.neurology.org/misc/addir.xhtml\#reprintsus

Neurol Neuroimmunol Neuroinflamm is an official journal of the American Academy of Neurology.

Published since April 2014, it is an open-access, online-only, continuous publication journal. Copyright

Copyright $\left({ }^{\circ} 2021\right.$ The Author(s). Published by Wolters Kluwer Health, Inc. on behalf of the American

Academy of Neurology.. All rights reserved. Online ISSN: 2332-7812.

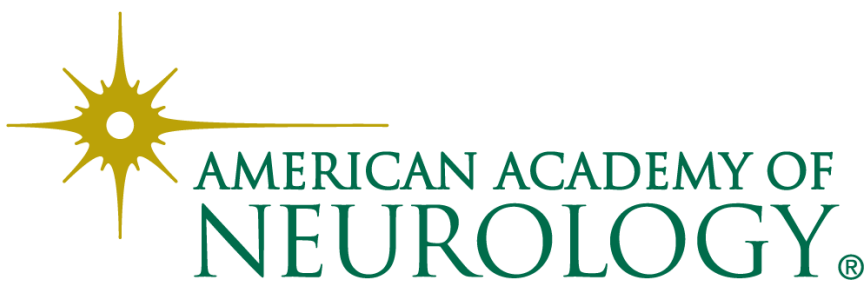

